\title{
Propuesta didáctica para la enseñanza de la derivación implícita.
}

Didactic proposed for the teaching of implicit differentiation

Proposta de ensino para o ensino da derivação implicado

\author{
Campo Antonio Nova García ${ }^{1}$ \\ Recibido: 15.09.2016 • Arbitrado: 18.10.2016 • Aprobado: 02.11.2016
}

\section{Resumen}

Este artículo parte de experiencias evaluativas con estudiantes de cursos universitarios en los que se detectaron errores en l a noción y manejo del concepto de derivación implícita y su aplicación al cálculo de pendientes de las rectas tangente y perpendicular a una curva en un punto dado. Se destaca el valor del aprendizaje significativo en la enseñanza actual de las matemáticas universitarias, en particular sobre estos tópicos y se utilizan conceptos avanzados dentro de las matemáticas para minimizar la ocurrencia de dichos errores. En la revisión teórica se utilizan varios referentes bibliográficos y se hace una sensibilización acerca de crear un mayor ambiente de seguridad que represente más confianza en el desempeño de los estudiantes universitarios.

Palabras clave: Derivación implícita, Recta tangente, Recta perpendicular, Aprendizaje Significativo.

\section{Abstract}

This article is evaluative experiences with students of university courses which were detected in other courses where errors are detected in the management notion and concept of implicit differentiation and its application to calculation of earrings. The value of meaningful lear-

${ }^{1}$ Ingeniero Electrónico, Universidad ENAP; Magíster en Administración, Universidad del Norte; Docente Universidad Eafit, cnovaga@eafit.edu.co 
ning in today's teaching college mathematics stands out, particularly on these topics and more advanced concepts are used in mathematics to minimize the occurrence of such errors. In the theoretical review several bibliographic references are used and sensitization is about creating a more secure environment representing more confidence in the performance of college students.

Keywords: Implicit differentiation, Tangent line, Perpendicular, Meaningful learning.

\section{Resumo}

Este artigo é baseado em experiências de avaliação com os alunos de cursos universitários em que os erros foram detectados no noção de gestão e conceito de diferenciação implícita e sua aplicação para o cálculo encostas da tangente, perpendicular a uma curva em linha reta em um ponto. $\mathrm{O}$ valor da aprendizagem significativa no ensino da matemática universitários de hoje destaca-se, em particular sobre estes temas e conceitos avançados são usados em matemática para minimizar a ocorrência de tais erros. Na revisão teórica várias referências bibliográficas são usados e sensibilização é sobre a criação de um ambiente mais seguro representando mais confiança no desempenho de estudantes universitários.

Palavras chave: Derivação implícita, tangente reta, perpendicular, aprendizagem significativa. 


\section{Introducción}

Impartir educación en matemáticas no es un trabajo fácil, particularmente en los primeros niveles de escolaridad. En el nivel universitario se facilita un poco, ya que la población objeto del aprendizaje tiene una inclinación voluntaria por las ciencias y particularmente por las Matemáticas (en el caso de la ingeniería) y de esta forma las diversas estrategias metodológicas que emplean los docentes para tal fin, pueden conseguir las metas propuestas para un grupo mayor de estudiantes, - con respecto a la secundaria- pero no para toda la población como se quisiera. En este sentido, los docentes profesionalizados en la enseñanza de este núcleo básico del conocimiento, salvaguardan unas técnicas y procedimientos concebidos como 'El Método', fundamento característico sobre el cual se basa el aprendizaje y con esta convicción buscan de manera permanente desarrollar tácticas y habilidades que garanticen una mejora sustantiva en el indicador que mide el número de estudiantes que realmente se apropian del conocimiento, «la matemática es, sobre todo, saber hacer, es una ciencia en la que el método claramente predomina sobre el contenido» (De Guzmán; 2007,p ).

\section{Estado del arte}

Dentro de los estudios y análisis más referenciados que se han elaborado en los últimos años sobre la enseñanza del Cálculo Diferencial, se presenta a continuación un resumen de los realizados, entre otros, por varias entidades estatales, universidades y grupos de investigación, que tocan el tema tangencialmente, pero que exploran casos similares en temas relacionados directamente. Se refieren algunos de los trabajos realizados por matemáticos con respecto a la enseñanza de noción y manejo de los conceptos de derivación en general y ecuaciones de las rectas tangente y perpendicular a una curva en un punto dado. Igualmente, se identifican algunas características restrictivas en la enseñanza de los tópicos en mención. 


\section{Antecedentes}

Es común usanza que las asignaturas: cálculo diferencial, integral y de varias variables, entre otras, sean presentadas a los estudiantes como materias abstractas que generan dificultad por sí mismas y esta conducta de entrada, aunada a los procedimientos matemáticos, acaban complicando a muchos alumnos y desviándolos del concepto que se está tratando, generándoles inseguridad y falta de confianza en lo aprendido. Autores diversos se han interesado por tales inconvenientes y han plasmado iniciativas para presentar los conocimientos analíticos de una forma más amena y posiblemente más comprensible para los educandos.

\section{La práctica en las universidades}

Los textos de Thomas (1979) y Leithold (1992), coinciden en la concepción que un curso de cálculo diferencial debe encaminarse a las aplicaciones del análisis invocando a la intuición, y luego, por el adiestramiento en el desarrollo de ejercicios, alcanzar la habilidad operacional. Hay otros textos que son referentes válidos de primer orden, como El Cálculo de Apóstol donde primero se estudia el cálculo integral y luego el cálculo diferencial, algo similar se puede apreciar en varias de universidades del mundo. En el extracto del prólogo a la primera edición (Apostol, 1967), el autor menciona lo siguiente:

La disposición de este libro ha sido sugerida por el desarrollo histórico y filosófico del Cálculo y la Geometría Analítica. Por ejemplo, se estudia la integración antes de la diferenciación. Aunque esta manera de ordenar la materia del curso sea poco frecuente, es históricamente correcta y pedagógicamente adecuada. Además, es el mejor camino para hacer patente la verdadera conexión entre la derivada y la integral (p. vii). 


\section{Propuesta didáctica para la enseñanza de funciones en el curso de cálculo diferencial de la Universidad Nacional de Colombia, sede Medellín}

De acuerdo con Mosquera (2013), uno de los temas de estudio fundamental y primordial en el proceso de enseñanza y aprendizaje en el área de las Matemáticas, es el de las derivadas, pues ellas constituyen una poderosa herramienta, de gran utilidad en el trabajo y estudio de los sistemas matemáticos. Adquirir habilidad para formular, modelar y resolver problemas que involucren procesos de diferenciación, resulta de suma importancia, por cuanto ello facilita la comprensión de nuestra realidad y da sentido a la enseñanza en diferentes contextos, no solo en las aplicaciones propias de las Matemáticas sino en áreas del conocimiento como las Ciencias Naturales, las Ciencias Sociales y muchas otras que establecen relación interdisciplinaria con las Matemáticas (Mosquera, 2013). Por otro lado, el trabajo con situaciones problema, posibilita el desarrollo del pensamiento lógico matemático de los estudiantes y de sus competencias. Formular y resolver problemas que involucren derivadas, deberá ir de la mano con la implementación de otros procesos generales tales como: modelación de situaciones de la realidad; comunicar; razonar; comparar y ejercitar procedimientos y algoritmos. Esta implementación deberá hacerse de manera activa, constructiva, dinámica y creativa, con el propósito de que los estudiantes realicen aprendizajes verdaderamente significativos.

\section{Libro - Taller para la enseñanza del concepto de derivada (un enfoque geométrico)}

Barrientos (2014), propone un Libro - Taller como herramienta directa del proceso de enseñanza aprendizaje de los estudiantes en el concepto de derivada, pensado para propiciar la reproducción y construcción del conocimiento. Esta propuesta interrelaciona, estructura y se proyecta como mediador directo, ordenado y continuo en el aprendizaje del concepto de derivada. Busca dinamizar e incrementar el desarrollo de las habilidades en los estudiantes respaldando la exploración, interacción y construcción de conocimientos. 


\section{La comprensión de la derivada como objeto de investiga- ción en didáctica de la Matemática}

Sánchez-Matamoros, García, \& Llinares (2008) disertan sobre la comprensión de la noción de derivada y las dificultades que presenta para los estudiantes de bachillerato y primeros años de cálculo en la Universidad. En dicho contexto, este trabajo revisa y organiza las aportaciones de las investigaciones realizadas sobre Matemática educativa para identificar el conocimiento generado y las áreas donde es necesario contribuir con información. La revisión se ha estructurado considerando lo que se conoce sobre la comprensión de la derivada de una función en un punto; el papel que desempeñan los sistemas de representación y las características distintivas del desarrollo de la derivada. Por último, se identifican líneas de investigación necesarias para aumentar nuestra comprensión de cómo los estudiantes dotan de significado y usan el concepto de derivada.

\section{Nociones analíticas sobre función cuadrática y la calculadora}

La idea del estudio (Carrillo et al., 2012), radica esencialmente en la exposición de la función cuadrática con base en la transformación de parámetros en el computador e inspeccionando con ello cómo varía la gráfica de la función. El autor, en el plano cartesiano, muestra las distorsiones que se generan cuando las escalas en los ejes no son las mismas, entonces, desarrolla un planteamiento sobre la conceptualización en general a partir del empleo de elementos determinados, utilizando la interpretación de gráficas como ayuda didáctica.

\section{Algunos errores comunes en cálculo}

Brousseau (1983), señala que los errores son a menudo el resultado de grandes concepciones inadecuadas acerca de aspectos fundamentales de las Matemáticas. Frecuentemente los errores se presentan como resultado de la aplicación correcta y crédula de un procedimiento imperfecto sistematizado, que se puede identificar con facilidad por el profesor. 
Por su parte, Rico \& Castro (1995) sostienen que también los errores pueden presentarse cuando el estudiante utiliza procedimientos imperfectos y posee concepciones inadecuadas que no son reconocidas por el profesor. Los alumnos con frecuencia inventan sus propias técnicas y métodos, no formales, pero altamente originales, para la realización de las tareas que se les proponen y la resolución de problemas. Mulhern (1989, citado por Rico, 1995), pone en evidencia que la mayor parte de los investigadores y especialistas coinciden en considerar dos características generales de los errores cometidos por los alumnos: los errores que surgen en la clase por lo general de una manera espontánea y los que sorprenden al profesor, aunque pueden haberse gestado desde mucho antes.

Así las cosas, se aprecia que los errores son recurrentes y específicos en cada estudiante. Son difíciles de superar porque demandan de una reorganización de los conocimientos en el alumno. Existe una preponderancia de los errores sistemáticos con referencia a los errores por casualidad. Los errores sistemáticos dejan ver los procesos mentales que han llevado al alumno a una comprensión equivocada. Los estudiantes en su momento no toman conciencia del error, pues no cuestionan lo que les parece obvio y no consideran el significado de los conceptos, reglas o símbolos con los que trabajan. Los errores sistemáticos son en general el resultado de concepciones inadecuadas de los fundamentos de la Matemática, identificable o no identificables por el profesor. Algunos errores se generan en el procesamiento que hace el alumno de la información que suministra el profesor. En algunos casos, los alumnos, por ejemplo, inventan su propio método y sus propias técnicas con base en el método explicado por el profesor.

\section{La derivación implícita}

La mayor parte de las funciones abordadas en los cursos de matemáticas de los primeros niveles universitarios pueden describirse expresando una variable explícitamente en términos de otra variable; por ejemplo,

$$
y=\sqrt{7 x^{6}-1} \text {, o bien: } y=x^{2} \cot x
$$


o, en general, $y=f(x)$. Sin embargo, algunas funciones se definen implícitamente por medio de una relación entre $x$ y $y$ como:

$$
\mathbf{3} x^{2}+\mathbf{3} y^{2}=300 \text { (1) o bien, } \quad x^{3}+y^{3}=6 x y
$$
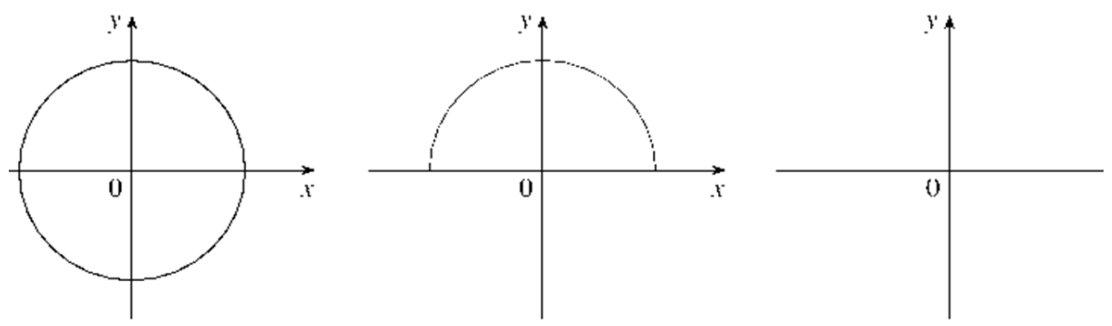

$$
\text { a) } x^{\uparrow} 2+y^{\uparrow} 2=100 \text { b) } f(x)=\sqrt{100-x^{2}} \quad \text { c) } g(x)=-\sqrt{100-x^{2}}
$$

Figura1. La circunferencia como relación puede contener dos o más funciones.

Fuente: Stewart (2010a).

No es una labor sencilla expresar $y$ en términos de $x$ a partir de la ecuación (2). Con CAS (sistema algebraico para computadora) no es difícil, pero las expresiones que se obtienen son muy complicadas. La gráfica de la ecuación (2) es una curva llamada folio de Descartes, ilustrada en la figura $2 \mathrm{y}$, de manera implícita, define a $y$ en términos de $x$ (Stewart; 2010a). 


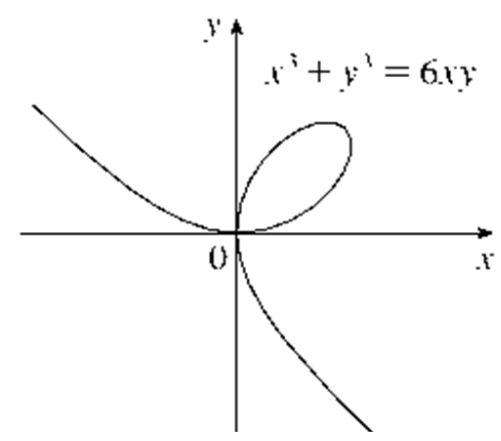

\section{Figura 2}

Folio de Descartes

Fuente: Stewart (2010a).

En la figura 3 se muestran las gráficas de esas tres funciones. Cuando se dice que $f$ es una función definida implícitamente por la ecuación (2), se da a entender que la ecuación

$$
x^{3}+[f(x)]^{3}=6 x f(x)
$$

es verdadera para todos los valores de $\mathrm{x}$ en el dominio de $f$.
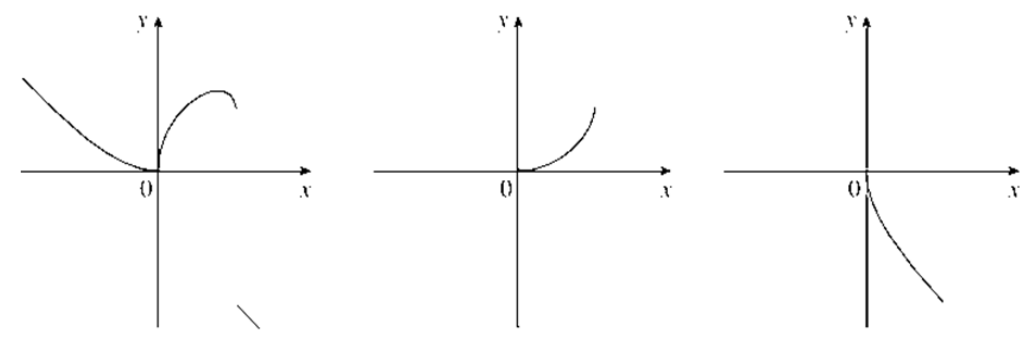

\section{Figura3}

Gráficas de tres funciones definidas por el

Folio de Descartes

Fuente: Stewart (2010a). 
No es necesario resolver una ecuación para $y$ en términos de $x$ con el fin de hallar la derivada de $y$. En lugar de ello, se aplica el método de derivación implícita. Este consiste en derivar ambos miembros de la ecuación con respecto a $x$, y a continuación, resolver la ecuación resultante para $y$. Sin embargo, es uno de los tópicos en los que más inseguridad presentan los estudiantes en las evaluaciones de cálculo diferencial (Stewart; 2010a).

Existen conceptos más avanzados que pertenecen al cálculo multivariable, que son fáciles de entender, que realmente no requieren prerrequisitos para manejarlos y que pueden ser una herramienta de comprobación de sus respuestas acertadas para generar una mayor confianza a los estudiantes de cálculo diferencial.

En los ejemplos y ejercicios siguientes, se supone que la ecuación dada determina $y$ implícitamente como una función derivable de $x$, de modo que puede aplicarse el método de derivación implícita.

\section{Primer ejemplo}

(a) Si $3 x^{2}+3 y^{2}=300$, encuentre $\frac{d y}{d x}$

(b) Encuentre la ecuación de la recta tangente y la recta perpendicular a la curva

$$
3 x^{2}+3 y^{2}=300 \text {, en el punto }(6,8) \text {. }
$$

\section{Solución 1 (implícita)}

(a) Primero simplificamos dentro de lo posible y derivamos en ambos miembros de la ecuación

$$
\frac{d}{d x}\left[x^{2}+y^{2}\right]=\frac{d}{d x}[100] \quad \rightarrow \frac{d}{d x}\left[x^{2}\right]+\frac{d}{d x}\left[y^{2}\right]=\frac{d}{d x}[100]
$$

Aquí hay que recordar que, $y$ es una función de $x$, entonces se aplica la regla de la cadena y obtenemos: 
$2 x(1)+2 y(d y / d x)=O$

Ahora, se despeja esta ecuación para $\frac{d y}{d x}=\frac{-x}{y}$

(b) En la coordenada $(6,8)$, se tienen los valores de $x=6$ y $y=8$, entonces verificamos que los valores del punto dado realmente satisfagan la igualdad:

$$
3 x^{2}+3 y^{2}=300 \rightarrow 3\left(6^{2}\right)+3\left(8^{2}\right)=300
$$

Con lo que se comprueba que el punto $(6,8)$ está sobre la circunferencia $x^{2}+y^{2}=100$

Ahora sí podemos reemplazar $(6,8)$ en la derivada obtenida para calcular la pendiente $m$, de la recta tangente a la circunferencia en el punto $(6,8)$

$$
m=\frac{d y}{d x}=\frac{-6}{\mathbf{8}}=\frac{-3}{\mathbf{4}}
$$

entonces, una ecuación de la tangente a la circunferencia $x^{2}+y^{2}=100$, en el punto $(6,8)$ es

$$
\frac{-3}{\mathbf{4}}=\frac{\Delta y}{\Delta x}=\frac{y-8}{x-6} \text { o en forma explícita: } y=\frac{-3 x}{4}+\frac{25}{2}
$$

Igualmente, el valor de la pendiente de la recta perpendicular a la circunferencia en $(6,8)$ es el inverso multiplicativo y negativo de la derivada en el punto en mención, esto es:

$$
m_{p}=-(m)^{-1} \rightarrow m_{p}=-\left(\frac{-3}{4}\right)^{-1}=\frac{4}{3}
$$

por lo tanto, una ecuación de la recta perpendicular a la circunferencia en $(6,8)$ es

$$
\frac{\mathbf{4}}{\mathbf{3}}=\frac{\Delta y}{\Delta x}=\frac{y-\mathbf{8}}{x-6} \text { o en forma explícita: } y=\frac{\mathbf{4} x}{\mathbf{3}}
$$


Ahora resolvemos nuevamente el ejercicio utilizando las funciones que definen a y, en forma explícita, en términos de $\mathrm{x}$. Al comparar las operaciones puede verse que el método de la derivación implícita ahorra una gran cantidad de trabajo, con respecto a la derivación explícita, así mismo, la derivación implícita funciona con igual facilidad para funciones más complejas para las cuales son imposibles de resolver para y en términos de x, como podrá apreciarse en las secciones 5. y 6 . En el ejemplo que hemos tomado, la ecuación se va a expresar respecto a una variable en términos de la otra, o sea de y en términos de $\mathrm{x}$.

\section{Solución 2 (explícita)}

(b) En la coordenada $(6,8)$, se tienen los valores de $x=6$ y $y=8$, entonces verificamos que el punto dado realmente satisfaga la igualdad

$$
3 x^{2}+3 y^{2}=300 \rightarrow 3\left(6^{2}\right)+3\left(8^{2}\right)=300
$$

Al despejar $y=f(x)$ en la ecuación $x^{2}+y^{2}=100$, se obtiene $y= \pm \sqrt{100-x^{2}}$

El punto $(6,8)$ se encuentra en la parte superior de la circunferencia $y$, por consiguiente, se considera la función $f(x)=\sqrt{100-x^{2}}$.

Aplicando la regla de la cadena a la función $f$, obtenemos:

$$
\begin{aligned}
f^{\prime}(x) & =\frac{\frac{1}{2\left(\left(100-\mathbf{x}^{2}\right)^{\frac{-1}{2}}\right) d y}}{d x} \\
\left(100-\mathbf{x}^{\dagger} 2\right) \rightarrow f^{\prime}(x)= & 1 / 2\left(\llbracket\left(100-\mathbf{x}^{\dagger} 2\right) \rrbracket^{\dagger}((-1) / 2)\right)(-2 x) \\
f^{\prime}(x) & =\frac{\frac{1}{2\left(\left(100-\mathbf{x}^{2}\right)^{\frac{-1}{2}}\right) d y}}{d x}
\end{aligned}
$$




$$
\left(100-\mathbf{x}^{\mathbf{\top}} 2\right) \rightarrow f^{\prime}(x)=(-\mathbf{x}) / \sqrt{ }\left(\llbracket 100-x \rrbracket^{\boldsymbol{\top}_{2}}\right)
$$

De modo que $f^{\prime}(6)=\frac{-6}{\sqrt{100-6^{2}}}=\frac{-3}{4}$ $y=\frac{-3 x}{4}+\frac{25}{2}$. la solución 1 , la ecuación de la tangente en $(6,8)$ es:

y la ecuación de la recta perpendicular será: $y=\frac{\mathbf{4} x}{\mathbf{3}}$

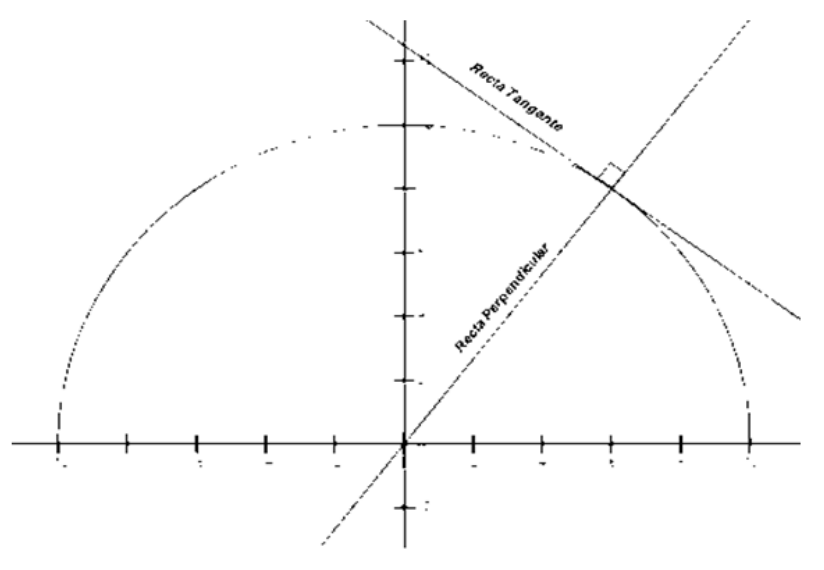

\section{Figura 4.}

Gráficas de la ecuación de la recta tangente y la recta perpendicular a la

Semicircunferencia $f(x)=\sqrt{100-x^{2}}$, en el punto $(6,8)$.

Fuente: Autor 
Segundo ejemplo

Encuentre $\frac{d y}{d x}$, si $x^{3}+y^{3}=6 x y$.

(b) Encuentre las ecuaciones de las rectas tangente y perpendicular al folio de Descartes $x^{\mathbf{3}}+y^{\mathbf{3}}=\mathbf{6} x y$, en el punto $(3,3)$.

Solución

(a) Si derivamos ambos miembros de la igualdad $x^{\mathbf{3}}+y^{\mathbf{3}}=\mathbf{6} x y$ con respecto a $\mathrm{x}$, considerando $y$ como función de $x$, y usamos la regla de la cadena en el término $y^{\mathbf{3}}$ y la regla del producto en el término $6 x y$, obtenemos:

$3 x^{2}+3 y^{2} y^{\prime}=6 x y^{\prime}+6 y$ o bien, $x^{2}+y^{2} y^{\prime}=2 x y y^{\prime}+2 y$

Ahora despejamos $\mathrm{y}^{\prime}: y^{2} y^{\prime}-2 x y^{\prime}=2 y^{-x^{2}}$

sacamos factor común en $y^{\prime} \frac{2 y-x^{2}}{y^{2}-2 x} \quad$ y despejamos: $y$ $\left(y^{2}-2 x\right)=2 y-x^{2} \rightarrow y^{\prime}=(i)$

(b) El punto $(3,3)$ satisface la igualdad $x^{3}+y^{3}=6 x y$, ya que: $27+27=(6)(9)$

Entonces: $\quad y^{\prime}=\frac{2(3) !(3)^{2}}{=-1}$

Una mirada a la Fig. 5 confirma que este es un valor razonable para la pendiente en $(3,3)$. En una recta con pendiente negativa, los valores de $y$ disminuyen a medida que aumentan en $x$. De este modo, una ecuación de la recta tangente al folio en $(3,3)$ es:

$-1=\frac{\Delta y}{\Delta x}=\frac{y-3}{x-3} \rightarrow x+y=\mathbf{6}$

y la pendiente de la recta perpendicular en el mismo punto será:

$m_{p}=-(m)^{-1} \rightarrow m_{p}=-(-1)^{-1}=1$ 
$1=\frac{\Delta y}{\Delta x}=\frac{y-\mathbf{3}}{x-\mathbf{3}}$ ocuación de la recta perpendicular en $(3,3)$ es:

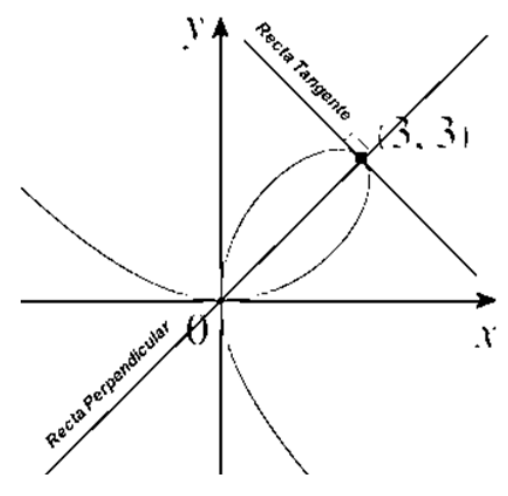

\section{Figura 5 .}

Gráficas de la ecuación de la recta tangente y la recta perpendicular a la curva "folio de Descartes"

Fuente: Stewart (2010a).

Solución Sencilla por derivadas parciales

Si suponemos que una ecuación de la forma $F(x, y)=0$ define $y$ implícitamente como una función derivable de x. (Larson \& Edwards, 2011). O sea: $y$ $=\mathbf{0}$. Para todo valor de $x$ que pertenezca a la función. Entonces si $\mathrm{F}$ es derivable, por regla de la cadena tenemos:

$$
\begin{aligned}
& \frac{d}{d x}[F(x, y)]=0 \rightarrow \frac{\partial F}{\partial x} \frac{d x}{d x}+\frac{\partial F}{\partial y} \frac{d y}{d x}=0 \\
& \rightarrow F x+F y \cdot f^{\prime}(x)=0 \rightarrow F x=-F y \cdot f^{\prime}(x) \rightarrow f^{\prime}(x)=\frac{-F x}{F y} \\
& =y^{\prime}
\end{aligned}
$$

Definiendo de esta forma el 'Teorema de la Regla de la Cadena para la Derivación Implícita', con una sencillez que se puede convertir en 
una estrategia para darle seguridad al estudiante que desarrolla ejercicios de derivación implícita en una variable e igualmente le hace ver que los temas que vienen en el cálculo de varias variables son manejables y una extensión de los actuales.

A continuación, se solucionan algunos ejemplos modelo que incluyen funciones diferentes y están planteados como ejercicios en los textos: Cálculo de Varias Variables de J. Stewart, Cálculo de Varias Variables de Zill y Wright, y Cálculo de Larson y Edwards:

Ejercicio 3.5-8 (Stewart, 2010a, p. 214). Encuentre $y^{\circ}$ en:

$$
y^{5}+x^{2} y^{3}=1+y e^{x^{2}}
$$

$F(x, y)=y^{5}+x^{2} y^{3}-1-y e^{x^{2}}=0 \rightarrow \mathbf{y}^{\prime}=\frac{-F x}{F y}=\frac{-\left(2 x y^{3}-2 x y e^{x^{2}}\right)}{5 y^{4}+3 x^{2} y^{2}-e^{x^{2}}}=$

$$
\frac{2 x y e^{x^{2}}-2 x y^{3}}{5 y^{4}+3 x^{2} y^{2}-e^{x^{2}}}
$$

Ejercicio 3.5-16 (Stewart, 2010a, p. 214). Encuentre $y^{\circ}$ en: $\operatorname{sen} x+\cos y=\operatorname{sen} x \cos y$

$$
\begin{aligned}
& F(x, y)=\operatorname{sen} x+\cos y-\operatorname{sen} x \cos y=0 \rightarrow y^{\prime}=\frac{-F x}{F y}=\frac{-(\cos x-\cos x \cos y)}{-\operatorname{sen} y+\operatorname{sen} x \operatorname{sen} y}= \\
& \frac{\cos x(\cos y-1)}{\operatorname{sen} y(\operatorname{sen} x-1)}
\end{aligned}
$$

Ejercicio 11.5-27. (Stewart, 2010b, p. 787). Encuentre y' en:

$$
\cos (x-y)=x e^{y}
$$

$$
\begin{aligned}
& F(x, y)=\cos (x-y)-x e^{y}=\mathbf{0} \rightarrow \mathbf{y}^{\prime}=\frac{-F x}{F y}=\frac{-\left[-\operatorname{sen}(x-y)-e^{y}\right]}{-\operatorname{sen}(x-y)(-1)-x e^{y}} \\
& \frac{\operatorname{sen}(x-y)+e^{y}}{\operatorname{sen}(x-y)-x e^{y}}
\end{aligned}
$$
Ejercicio 13.5-18 (Zill \& Wright, 2011, p. 716). Halle $\frac{d y}{d x}$ en la ecua-
ción $x+2 y^{2}=e^{y}$

$$
F(x, y)=x+2 y^{2}-e^{y}=0 \rightarrow y^{\prime}=\frac{-F x}{F y}=\frac{-1}{4 y-e^{y}}=\frac{1}{e^{y}-4 y}
$$


Ejercicio 13.5-19 (Zill \& Wright, 2011, p. 716). Halle $\frac{d y}{d x}$ en la ecuación $\mathrm{y}=\operatorname{sen} x y$

$F(x, y)=y-\operatorname{sen} x y=0 \rightarrow y^{\prime}=\frac{-F x}{F y} \rightarrow y^{\prime}=\frac{-(y \cos x y)}{1-x \cos x y} \rightarrow y^{\prime}=\frac{y \cos x y}{1-x \cos x y}$

Ejercicio 13.5-27 (Larson \& Edwards, 2011, p. 931). Halle $\frac{d y}{d x}$ en la ecuación

$$
\begin{gathered}
x^{2}-x y+y^{2}-x+y=0 \rightarrow F(x, y)=x^{2}-x y+y^{2}-x+y=0 \\
y^{\prime}=\frac{-F x}{F y} \rightarrow y^{\prime}=\frac{-(2 x-y-1)}{-x+2 y+1} \rightarrow y^{\prime}=\frac{y-2 x+1}{2 y-x+1}
\end{gathered}
$$

Solución larga por derivación implícita

Ejercicio 3.5-8 (Stewart, 2010a, p. 214). Encuentre $y^{*}$ en:

$$
y^{5}+x^{2} y^{3}=1+y e^{x^{2}}
$$

$\llbracket 5 y^{4} y^{\prime}+(2 x)\left(y^{3}\right)+\left(3 y \rrbracket^{2} y^{\prime}\right)\left(x^{2}\right)=0+y^{-}\left(e^{x^{2}}\right)+\left(e^{x^{2}}\right)(2 x) y$

$5 y^{4} y^{\prime}+3 x^{2} y^{2} y^{\prime}-e^{x^{2}} y^{\prime}=2 x y e^{x^{2}}-2 x y^{3} \rightarrow \llbracket y^{\prime}\left(5 y \rrbracket^{4}+3 x^{2} y^{2}-e^{x^{2}}\right)=2 x y e^{x^{2}}-2 x y^{3}$

$y^{\prime}=\frac{2 x y e^{x^{2}}-2 x y^{3}}{5 y^{4}+3 x^{2} y^{2}-e^{x^{2}}}$

Ejercicio 3.5-16 (Stewart, 2010a, p. 214). Encuentre $y^{\circ}$ en: $\operatorname{sen} x+\cos y=\operatorname{sen} x \cos y$

$$
\begin{aligned}
& \cos x-(\operatorname{sen} y)\left(y^{\prime}\right)=\cos x \cos y+(-\operatorname{sen} y)\left(y^{\prime}\right) \operatorname{sen} x \\
& -(\operatorname{sen} y)\left(y^{\prime}\right)+(\operatorname{sen} x \operatorname{sen} y)\left(y^{\prime}\right)=\cos x \cos y-\cos x \\
& y^{\prime}(\operatorname{sen} x \operatorname{sen} y-\operatorname{sen} y)=\cos x(\cos y-1) \\
& y^{\prime}=\frac{-(\cos x-\cos x \cos y)}{-\operatorname{sen} y+\operatorname{sen} x \operatorname{sen} y} \rightarrow y^{\prime}=\frac{\cos x(\cos y-1)}{\operatorname{sen} y(\operatorname{sen} x-1)}
\end{aligned}
$$


Ejercicio 11.5-27. (Stewart, 2010b, p. 787). Encuentre $y^{*}$ en:

$$
\begin{aligned}
& \cos (x-y)=x e^{y} \\
& -\operatorname{sen}(x-y)\left(1-y^{\prime}\right)=1\left(e^{\boldsymbol{\top}} y\right)+x\left(e^{\boldsymbol{\top}} y y^{\circ}\right) \rightarrow-\operatorname{sen}(x-y)\left(1-y^{\prime}\right) \\
& =e^{y}+x e^{y} y^{-} \\
& -\operatorname{sen}(x-y)+\operatorname{sen}(x-y) y^{\circ}=
\end{aligned}
$$

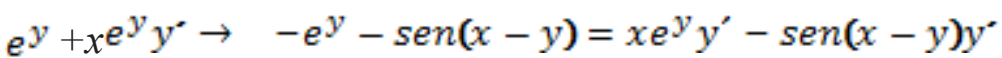

$$
\begin{aligned}
& -e^{y}-\operatorname{sen}(x-y)=y^{-}\left[x e^{y}-\operatorname{sen}(x-y)\right] \\
& y^{\prime}=\frac{-e^{y}-\operatorname{sen}(x-y)}{x e^{y}-\operatorname{sen}(x-y)} \rightarrow y^{\prime}=\frac{-\left[\operatorname{sen}(x-y)+e^{y}\right]}{-\left[\operatorname{sen}(x-y)-x e^{y}\right]} \\
& \rightarrow y^{\prime}=\frac{\operatorname{sen}(x-y)+e^{y}}{\operatorname{sen}(x-y)-x e^{y}}
\end{aligned}
$$

Ejercicio 13.5-18 (Zill \& Wright, 2011, p. 716). Halle $\frac{d y}{d x}$ en la ecuación $x+2 y^{2}=e^{y}$

$$
\begin{aligned}
& x+2 y^{2}-e^{y}=0 \rightarrow 1+4 y y^{\prime}-e^{y} y^{\prime}-\cap \\
& 1=e^{y} y^{\prime}-4 y y^{\prime} \rightarrow 1=y^{\prime}\left(e^{y}-4 y\right)
\end{aligned} \rightarrow y^{\prime}=\frac{1}{e^{y}-4 y}
$$

Ejercicio 13.5-19 (Zill \& Wright, 2011, p. 716). Halle $\frac{d y}{d x}$ en la ecuación $y=\operatorname{sen} x y$

$$
\begin{aligned}
& y-\operatorname{sen} x y=0 \rightarrow y^{\prime}-(\cos x y)\left(1 * y+y^{\prime} * x\right)=0 \\
& \quad y^{\prime}-\cos x y\left(y+x y^{\prime}\right)=0 \rightarrow y^{\prime}-y \cos x y-(x \cos x y) y^{\prime}=\mathbf{0} \\
& \rightarrow y^{\prime}-(x \cos x y) y^{\prime}=y \cos x y \rightarrow y^{\prime}(1-x \cos x y)=y \cos x y \\
& \rightarrow y^{\prime}=\frac{y \cos x y}{1-x \cos x y}
\end{aligned}
$$

Ejercicio 13.5-27 (Larson \& Edwards, 2011, p. 931). Halle $\frac{d y}{d x}$ en la ecuación

$$
\begin{aligned}
& x^{2}-x y+y^{2}-x+y=0 \\
& 2 x-\left(1 * y+1 * y^{\prime} * x\right)+2 y y^{\prime}-1+y^{\prime}=0
\end{aligned}
$$




$$
\begin{aligned}
& 2 x-y-x y^{\prime}+2 y y^{\prime}-1+y^{\prime}=0 \rightarrow 2 x-y-1=x y^{\prime}-2 y y^{\prime}-1-y^{\prime} \\
& 2 x-y-1=y^{\prime}(x-2 y-1) \rightarrow y^{\prime}=\frac{2 x-y-1}{x-2 y-1} \\
& y^{\prime}=(-(y-2 x+1)) /\left(-(2 y-x+1) \rightarrow y^{\prime}=(y-2 x+1) /(2 y-x+1)\right.
\end{aligned}
$$

\section{Comparación del método expuesto en la sección 5. con el de la sección 6}

En el primer método (Sección 5.) se asumen $x$ e $y$ como variables independientes a diferencia del segundo método (Sección 6.) en el cual en todo momento debe tenerse en cuenta que $y$ es función de $x$.

El hecho de que, $y$ no dependa de $x$, hace que se disminuya el número de veces en los que se utiliza la regla de la cadena y esto, a su vez, disminuye el número total de operaciones en el procedimiento.

El número de operaciones disminuye aún más, en el primer método (Sección 5.), debido a que no es necesario despejar $y^{\prime}$. El cociente que se calcula, $-F x / F y$, entrega directamente la expresión para $\mathrm{y}^{\prime}$.

De esta manera, el método expuesto puede estructurarse como una técnica adicional de comprobación previa para obtener la respuesta correcta de $y^{\prime}$. 


\section{Conclusiones}

En el estudio de las Matemáticas, ha sido común recurrir a artilugios para crear un mayor ambiente de seguridad y confianza en los aprendices, como saber lo que voy a obtener en un límite mediante la fórmula de derivación, la confirmación anticipada en el método de integración por fracciones simples, la transformada de Laplace y la misma regla de L'Hôpital, entre muchas.

El hecho de que haya textos y universidades, que son referentes reconocidos donde primero se estudia el cálculo integral y luego el cálculo diferencial, ponen de manifiesto que al margen de los procesos de movilidad académica, al parecer no existe un acuerdo definitivo sobre lo que ha de componer un primer curso de cálculo, entonces podemos repensar la enseñanza con formas de comprobación para los alumnos, recurriendo a la simplicidad de temas en cursos más avanzados de cálculo, en forma tal que podamos mejorar el desempeño de los estudiantes universitarios, generar una mayor sensación de seguridad y a su vez minimizar la ocurrencia de errores, garantizando de alguna manera un mayor nivel de éxito y un aprendizaje más cercano a lo significativo.

Aunque el docente resuelva trabajar una sola manera de desarrollar ejercicios o se ajuste con un determinado texto guía, es conveniente intentar y describir otros alternos para cada una de ellos, cotejarlos con el que se desarrollan en clase y estimar sus ventajas y desventajas.

Todo intento que realice el docente de ciencias básicas con el propósito de lograr una mejoría en el desempeño de sus educandos en su proceso de aprendizaje tiene plena vigencia siempre que sea factible, aceptable y conducente, buscando que los alumnos dominen por completo las soluciones correctas de los ejercicios; de esta forma tendrán más tiempo para dedicarse a trabajar en los aspectos nuevos o inexplorados del tema al que se enfrentan.

El tiempo de preparación de cada una de las clases por parte de los docentes, es una labor permanente de la cual el docente no se puede sustraer, este debe preocuparse por sus alumnos, ser innovador y 
creativo, buscando siempre la manera de involucrar cada vez más estudiantes en la apropiación del conocimiento. Al educador no le conviene tornarse indiferente y menos despreocupado por un bajo rendimiento de alguno de sus alumnos, por el contrario, debe sustentarse de ese deseo de lograr transmitir su conocimiento de una manera eficiente y eficaz y del amor por su labor docente. 


\section{ReferenciasBibliográfica}

Apostol, T. (1967). One-Variable Calculus, with an Introduction to Linear Algebra Vol. I. 2nd. Edition. New York: John Wiley \& Sons., p. vii.

Barrientos, P. (2014). Libro-taller para la enseñanza del concepto de derivada. Medellín: Universidad Nacional de Colombia.

Brousseau, G. (1983). Los obstáculos epistemológicos y los problemas en Matemáticas. Disponible en http://fractus.mat.uson.mx/Papers/Brousseau/ObstaculosBrousseau.htm

Carrillo, A. et al. (2012). TIC's: Algunas ideas sobre la función cuadrática y calculadora. Revista electrónica: Revista Iberoamericana de Educación Matemática Número 32. Disponible en http://www.fisem.org/www/union/revistas/2012/32/archivo14volumen32.pdf.

De Guzmán, M. (2007). Enseñanza de las Ciencias y la Matemática. Revista Iberoamericana de Educación. 43 (19-58). Disponible en http://dialnet.unirioja. es/ejemplar/159597

Larson, R., Edwards, B. (2011). Cálculo. Novena Edición. México, D. F.: McGraw-Hill/ Interamericana Editores, S.A. 95, 141-142, 749.

Leithold, L. (1992). El Cálculo. 7a. Edición. México, D. F.: Editorial Oxford - Harla. 172-182. Mosquera, A. (2013). Propuesta Didáctica para la Enseñanza de Funciones en el Curso de Cálculo Diferencial de la Universidad Nacional de Colombia, Sede Medellín. Trabajo presentado para optar al título de: Magíster en Enseñanza de las Ciencias Exactas y Naturales. Universidad Nacional de Colombia - Sede Medellín.

Rico, L. (1995). Errores y dificultades en el aprendizaje de las Matemáticas, cap. 3. pp. 69-108, En Kilpatrik, J.; Gómez, P., y Rico, L.: Educación Matemática. México: Grupo Editorial Iberoamérica.

Sánchez-Matamoros, G., García, M., Llinares, S. (2008). La Comprensión de la Derivada como Objeto de Investigación en Didáctica de la Matemática. Revista 
latinoamericana de investigación en matemática educativa Relime, vol.11, No.2, pp.21-22. Disponible en http://www.scielo.org.mx/scielo.php?pid=S166 5-24362008000200005\&script=sci arttext, ISSN 2007-6819.

Stewart, J. (2010). Cálculo de una Variable, conceptos y contextos. $4^{\mathrm{a}}$. Edición. México, D. F.: Cengage Learning Editores S.A., 209-216

Thomas, G. Jr. (1979). Cálculo Infinitesimal y Geometría Analítica. 6ª . Edición. Madrid: Aguilar S.A. de Ediciones, 114-118.

Zill, D., Wright, W. (2011). Cálculo de Varias Variables. 4ª Edición. México, D. F.: Mc Graw Hill, 711-716. 


\section{Instrucciones para los autores}

\section{Tipo de artículo}

La revista actualmente hace énfasis en la publicación de documentos que se asimilen a la tipología exigida por el Servicio Permanente de Indexación de Revistas Científicas y Tecnológicas Colombianas, y de manera especial los correspondientes a las tipologías 1 y 2:

Artículo de investigación científica y tecnológica: documento que presente, de manera detallada, los resultados originales de proyectos de investigación. La estructura general del articulo debe contener los siguientes apartados:

Resumen: especifica de una manera corta, pero clara y concisa, los siguientes ítems: introducción, objetivos, metodología, principales resultados y conclusiones del estudio.

Introducción: se describe el problema de investigación, justificación y antecedentes del tema investigado.

Método: se describe la metodología empleada en la investigación.

Fundamentación teórica: se exponen principales referentes teóricos utilizados.

Resultados o hallazgos, incluyendo su discusión.

Conclusiones y recomendaciones: deben presentar una respuesta clara a los interrogantes que género la investigación y corresponder a los objetivos planteados. Es conveniente indicar las limitaciones que se presentaron en el estudio, al igual como logros y posibles aplicaciones de resultados.

Las referencias bibliográficas al final y a pie de página, al igual como referencias en el texto, deben hacerse siguiendo las normas APA. 
1. Artículo de revisión: documento resultado de una investigación documental en el cual se analizan, sistematizan e integran resultados de investigaciones publicadas o no publicadas, sobre un campo en ciencia o tecnología, con el fin de dar cuenta de los avances y las tendencias de desarrollo. Se caracteriza por presentar una cuidadosa revisión bibliográfica de por lo menos, 50 referencias.

\section{Recepción de artículos}

Para la preparación de los manuscritos deben seguirse las normas de publicación de la APA versión 6. La extensión no debe exceder las 6000 palabras. Se admite máximo 5 figuras y/o 5 tablas.

Para enviar el artículo debe ingresar a la plataforma en línea Open Journal System en la dirección http://revistas.iue.edu.co/index.php/ katharsis/index. Allí se registrará como autor asignando un nombre de usuario y contraseña y siga los pasos que allí se indican para subir su artículo. Inmediatamente, se notificará la recepción y el código que le ha sido asignado a su manuscrito. Para mayor información se puede comunicar al correo katharsis@iue.edu.co

\section{Normas eticas de publicacion}

\subsection{Declaración y sometimiento}

Los artículos enviados para su publicación deben ser textos originales. Se debe anexar una carta de sometimiento del artículo y cesión de derechos la cual indica que el artículo no ha sido publicado previamente ni enviado simultáneamente a otra revista o medio de difusión, y que todos los autores han leído y están de acuerdo con su contenido y están concediendo a la revista Katharsis el derecho para que su artículo y materiales sean reproducidos, publicados, editados, comunicados y trasmitidos públicamente en cualquier forma o medio, así como su distribución en el número de ejemplares que se requieran y su comunicación pública, incluida su puesta a disposición del público nacional e internacional a través de medios electrónicos, para fines exclusivamen- 
te científicos, culturales y de difusión y sin fines de lucro. Es aceptable publicar un artículo en otro idioma, indicando debidamente la fuente original del trabajo.

Es necesario tener presente que el estudio del cual se deriva el manuscrito debe contar con los consentimientos informados para todos los participantes y el respaldo del comité de ética institucional. Lo anterior debe especificarse en el apartado de metodología, al igual como las respectivas referencias en caso de contar con datos o pruebas proporcionadas o diseñadas por otros autores.

Los respectivos autores del artículo se hacen responsables del contenido del texto y de su efectiva participación como autores del texto presentado.

Será muy útil para facilitar el proceso de revisión que en el momento del envío del artículo el autor proporcione en la carta, información sobre por lo menos dos posibles revisores para su artículo (nombre, institución y correo electrónico).

\subsection{Autoría}

La revista Katharsis, partiendo de los cuatro criterios asumidos por el International Committee of Medical Journal Editors (ICMJE), asume la siguiente definición de autor de un artículo científico: 1. Realizar contribuciones sustanciales a la concepción o el diseño del articulo o la adquisición, análisis e interpretación de los datos para el trabajo; 2. La redacción del artículo o la revisión crítica de su contenido; 3. La aprobación final de la versión para publicar; 4. Ser responsable de todos los aspectos del trabajo, garantizando que todas las cuestiones relativas a la exactitud o integridad del texto sean investigadas y confirmadas. Solo quienes cumplen con estos 4 criterios pueden aparecer como autores de un artículo científico. Lo anterior implica que todos los coautores tengan una responsabilidad compartida de toda la obra en su conjunto, contando con la confianza en la integridad de contribuciones de sus colaboradores. 
Los contribuyentes al artículo que no cumplen con estos 4 criterios no pueden aparecer como autores, sino que deben ser reconocidos, indicando al pie de página sus nombres y la respectiva contribución realizada para los fines de la investigación o realización del artículo.

Es la responsabilidad colectiva de los autores, y no de la revista, determinar que todas las personas nombradas como autores cumplen los cuatro criterios. Los mismos autores del artículo en corresponsabilidad mutua deciden el orden de su aparición en el artículo según la importancia de sus contribuciones respectivas. Junto con el artículo a la revista se envía un formato de remisión del artículo, donde los autores (en caso de que sean más de uno), presentan sus datos en el orden establecido, del cual se hacen responsables.

Si los autores solicitan la eliminación o adición de un autor después de la presentación o la publicación del manuscrito, la revista solicitará una declaración firmada de acuerdo para el cambio solicitado de todos los autores mencionados y del autor debe ser eliminado o añadido.

\subsection{Plagio}

El plagio es un fenómeno bastante amplio y puede variar desde la ausencia de referencias bibliográficas, hasta el uso de ideas de otras personas, tanto publicadas, como sin publicar, sin su consentimiento, hasta la presentación de un documento completo con una autoría diferente en otro idioma. Para evitar el plagio, todas las fuentes deben citarse de forma apropiada según normas APA, y cuando se requiere el uso de gran cantidad de material escrito de otra persona o ilustraciones, debe solicitarse una autorización. Los procedimientos y pruebas utilizados en la investigación, que sean creados por otras personas, deben citarse explícitamente en el artículo. Si se detecta plagio en un artículo, este se retira inmediatamente de su proceso de evaluación, edición o publicación y se envía una carta a los autores con exposición de evidencias. 


\subsection{Retractación}

Las retractaciones se realizan por errores voluntarios o involuntarios de los autores; sin embargo, cualquiera que sea la causa, las acciones correctivas son iguales para ambas. Los autores tienen derecho a la retractación manifiesta y espontánea del contenido parcial o total de sus manuscritos en cualquier momento. La comunicación en donde se retracte debe ser suficientemente explícita. No excluye a los autores de las acciones disciplinarias o legales derivadas de los errores de los artículos

\section{Presentación de artículos}

\subsection{Encabezamiento}

\subsubsection{Título}

Lo más corto posible, sin interrogaciones ni exclamaciones y con carácter afirmativo. Se recomienda escribirlo con un máximo de 15 palabras; en caso de no poder hacerse así, conviene dividirlo en título y subtítulo, separados por dos puntos. Debe dar cuenta del tema y adelantar la conclusión. Además del título en español, debe incluirse el título en inglés. En el caso de que el título requiera alguna explicación en pie de página, ésta se referencia con un asterisco escrito inmediatamente después del título.

Se debe especificar si el artículo enviado es producto de una investigación, tesis de grado o ensayo. Si es un producto investigativo, debe establecerse el nombre del proyecto, la institución que lo apoya y el código de registro (si lo tiene); si es producto de un trabajo de tesis debe constar el título académico al que se optó y el título o tema general de la misma. 


\section{Información sobre los autores}

Comprende la formación académica de los autores: último título obtenido, universidad que lo otorgó.

Debe establecerse su filiación institucional: entidad en la que trabajan, ciudad, país, dirección de correo electrónico.

\section{Resumen y palabras clave}

Después del título y del nombre de los autores se debe elaborar un resumen analítico, seguido de las palabras clave. El resumen debe ser escrito sin sangría, en tercera persona y en tiempo presente; debe presentarse con la siguiente estructura: objetivo, metodología, resultados, discusión y conclusiones; debe escribirse en idioma español y en inglés (Abstract). El resumen debe oscilar entre 200 y 250 palabras.

Las palabras clave deben oscilar entre 4 y 6 , estas deben evidenciar los temas y conceptos principales del artículo, de acuerdo con los tesauros especializados. Tanto el título, como el resumen y las palabras clave deben ser traducidos al inglés.

Se recomienda normalizar las palabras claves que son aceptadas por bases de datos internacionales. Pueden ser consultadas en los siguientes enlaces:

Biblioteca virtual en Salud de la OPS, disponible en: http://www. bvsalud.org

Descriptores en ciencias de la salud, a través del enlace DeCS-Terminología en salud, disponible en: http://decs.bvs.br/E/homepagee.htm

Tesauro de la UNESCO: http://databases.unesco.org/thessp/

Para palabras en inglés: http://www.ncbi.nlm.nih.gov/mesh

\section{Cuerpo del artículo}

Si el artículo da cuenta de investigaciones científicas, debe contener, además de las partes antes mencionadas: 
Introducción, métodos, resultados, discusión, conclusiones y, según el caso, recomendaciones. Los estudios de casos deben acoger los aspectos metodológicos.

Estas mismas secciones aplican para investigación cualitativa y revisiones de tema.

Para otros tipos de artículo el texto se podrá desarrollar sin guardar estructura.

\section{Introducción}

Contiene el desarrollo y los antecedentes del problema. Será breve y deberá proporcionar sólo la explicación necesaria para que el lector pueda comprender el texto que sigue a continuación. No debe contener tablas ni figuras, a menos que sean imprescindibles para la comprensión del texto. Debe incluir un último párrafo en el que se exponga de forma clara el o los objetivos del trabajo.

\section{Metodología}

Se describirá claramente la selección de la población identificando el sexo, la edad y otras características importantes. Se debe especificar cómo se recolectaron los datos. Se hará referencia al tipo de estudio y tipo de análisis empleados (descriptivo, epidemiológico, experimental, ensayo clínico, etc), el sistema de asignación de los sujetos. Identificar los métodos, instrumentos de evaluación, tratamientos, fármacos o productos químicos, indicando su nombre genérico, dosificación y vía de administración. Indicar el paquete estadístico utilizado.

Cuando se trate de trabajos experimentales realizados con animales o humanos, debe indicarse explícitamente que se han seguido las normas éticas de investigación, y que se han cumplido los convenios internacionales de experimentación animal o humana (Declaración de Helsinki, disponibleen: http://www.wma.net/es/3opublications/1opolicies/b3/). Asimismo manifestar que la investigación fue aprobada por la comisión de ética de la institución donde la investiga- 
ción fue realizada e indicar si se obtuvo consentimiento informado por escrito por parte de las personas que participaron en la investigación.

\section{Resultados}

Los resultados deben presentarse en una secuencia lógica, en textos apoyados en tablas y figuras que expresen claramente los resultados del estudio. No repita en el texto todos los datos de las tablas y figuras, sólo los más importantes. Enfatice y resuma sólo las observaciones más importantes.

\section{Discusión y conclusiones}

Enfatice los aspectos nuevos e importantes del estudio y las conclusiones que se derivan del mismo. No repita en detalle los resultados que ha presentado en la sección anterior ni en la introducción. Relacione lo obtenido con otros estudios relevantes sobre el tema. Indique las implicaciones de sus hallazgos y sus limitaciones. Relacione las conclusiones con los objetivos del estudio. No haga suposiciones si no se ven apoyadas por los datos. Cuando sea apropiado puede incluirse recomendaciones.

\section{Agradecimientos}

Cuando se considere necesario se citará a las personas, centros o entidades que hayan colaborado o apoyado la realización del trabajo.

\section{Referencias bibliográficas}

La citación de las referencias bibliográficas deberá realizarse al final del artículo bajo el título Referencias; éstas incluyen solo las fuentes que sustentan la investigación y que se utilizaron para la preparación del trabajo y que han sido citadas en el cuerpo del texto, a diferencia de la bibliografía que incluye las fuentes que sirven para profundizar en el tema. De acuerdo con las normas APA, las referencias se elaboran 
conforme a las siguientes consideraciones: nombres completos, orden alfabético por la primera letra de la referencia; las obras de un mismo autor se ordenan cronológicamente, con interlineado doble. Si hay varias obras de un mismo autor y de un mismo año, se diferencian unas de otras agregando al año una letra del alfabeto.

Citas de referencia en el texto. Las referencias bibliográficas se harán en el cuerpo del texto, según establecen las normas APA: apellido del autor, año de publicación y página, según sea el caso.

Ejemplos para citar en el texto una obra por un autor(a):

Rivera (1994) comparó los tiempos de reacción...

En un estudio reciente sobre tiempos de reacción (Rivera, 1994)...

En 1994, Rivera comparó los tiempos de reacción...

\section{Referencia de libro:}

Autor, A.A. (año de publicación). Título de la obra. (Edición-si la hay). Ubicación: Editorial.

Ejemplo:

López, D. (2006). El derecho de los jueces. Bogotá: Universidad de los Andes; Legis.

\section{Capítulo de un libro:}

Autor, A.A., \& Autor, B.B. (Año de la publicación). Título del capítulo. A. Editor \& B. Editor (Eds.), Título del libro (páginas del capítulo). Ubicación: Editor.

Ejemplo:

Létourneau. J. (2009). Como documentarse en la era electrónica. 
Guía de iniciación al trabajo intelectual. La caja de herramientas del joven investigador (pp. 35-76). Medellín: La carreta editores.

Avendaño M., L. F. (2009). Correlación y diferencias entre el derecho penal y el derecho disciplinario a la luz del principio del non bis in ídem. Medellín: Universidad de Antioquia.

\section{Referencias Sentencias y Normativa:}

Colombia (2002). Código Disciplinario Único, Ley 734.

Colombia. Corte Constitucional (1993). Sentencia C-417 de 1993. Magistrado Ponente: José Gregorio Hernández Galindo.

Colombia. Corte Constitucional (1994). Sentencia T-254 de 1994. Magistrado Ponente: Eduardo Cifuentes Muñoz.

Colombia. Corte Constitucional (1995). Sentencia T-007 de 1995. Magistrado Ponente: Antonio Barrera Carbonell.

\section{Cita de revista:}

Dworkin, R. (2007). ¿Deben nuestros jueces ser filósofos?, ¿̇pueden ser filósofos? (Trd. García, L.). En: Estudios de Derecho, No. 144, año LXIV, U. de A., Medellín.

\section{Cita de Internet:}

Alfonso, S. (2008). Neoconstitucionalismo. Anales de la Academia de Ciencias Morales y Políticas, Recuperado http://ancmyp.org.ar/ user/files/o2neoconstitucionalismo.pdf.

\section{Material gráfico}

Los Cuadros y Figuras deberán citarse explícitamente en el texto del artículo, e insertarse siempre a continuación del fin de párrafo donde se hace la cita por primera vez; no ponerlas todas al final de las secciones o del artículo, ni cortar un párrafo para insertarlas. Las figuras 
deben tener óptima resolución y los cuadros deben estar en formato editable, no como imagen.

La numeración es única y secuencial, sin importar la sección. Se usará entonces: Figura 1, Figura 2, etc,. Cuadro 1, Cuadro 2, etc. La segunda vez que se cite una Figura o Cuadro, esta ya debe haber sido previamente incluida.

Todas las Figuras y Cuadros irán precedidas de un espacio, deberán ir centradas, tituladas en la parte inferior, seguidas de un espacio. Cuando las figuras o Tablas son copiadas de otra fuente, ésta se debe citar tanto en el texto, como en la parte inferior derecha de la misma. Si son tablas propias, pero las cifras son tomadas de otra fuente, en la reseña se reemplaza "tomado de: "por "Fuente: ".

En las figuras se debe escribir en cada eje las variables (NO usar siglas!) y sus unidades; Ejemplo: Temperatura $\left({ }^{\circ} \mathrm{C}\right)$. Cuando sean varias gráficas se debe indicar el parámetro asociado a cada una de ellas. En los Cuadros, se procede igual para cada columna y fila, cuando sea del caso.

\section{Proceso evaluación}

La recepción del artículo en la revista no significa su aprobación. Los originales recibidos son sometidos a un proceso editorial que se desarrolla en varias fases. En primer lugar, los artículos pasan por una evaluación preliminar por parte de los miembros del Comité Editorial, quienes determinan la pertinencia de su publicación. Una vez establecido que el artículo cumple con requisitos temáticos y formales exigidos por la revista, será enviado a dos pares académicos, uno interno y otro externo a la Institución Universitaria de Envigado. En caso del concepto favorable emitido por árbitros se inicia el proceso editorial de los artículos en el orden de su recepción. El material enviado a la revista y no publicado no será devuelto a los autores.

Los pares son nombrados por el Comité Editorial y se sujetan en su evaluación al formato enviado para tal fin. Los autores pueden sugerir posibles evaluadores, sin embargo, la decisión final sobre los árbitros 
será tomada por el Comité Editorial de la revista. El proceso de evaluación es doble ciego, con el fin de evitar todo tipo de conflicto de intereses. Los casos de discrepancia entre dos evaluadores son valorados por el Comité Editorial que decide, finalmente, la posibilidad de la publicación. Los resultados del proceso de evaluación por pares académicos serán inapelables en todos los casos.

Los pares evaluadores considerarán los siguientes aspectos de los artículos enviados:

- Coherencia en el título, objetivos, hipótesis, método, resultados y discusión en el estudio presentado.

- Pertinencia de la fundamentación teórica.

- Calidad de redacción.

- Actualidad y relevancia de la bibliografía.

- Seguimiento de normas de citación (APA).

- Originalidad del estudio e importancia de aportes al respectivo campo de conocimiento.

El resultado de la evaluación puede ser: 1) Publicar sin cambios; 2) Publicar cuando se hayan realizado las correcciones menores; 3) Publicar cuando se haya efectuado una revisión a fondo y 4) Rechazar.

En caso de que sean requeridos ajustes al artículo, la versión corregida debe ser devuelta a la revista en un tiempo no mayor a 15 días.

Una vez el escrito sea aceptado por pares para su publicación, se inicia el proceso de su edición, incluyendo revisión de estilo, de redacción $\mathrm{y}$ otros aspectos formales.

Durante todo el proceso el editor mantiene una comunicación con el autor remitente con el fin de solicitar ajustes requeridos y avisar sobre el proceso editorial.

Una vez publicado el número respectivo, se emite la comunicación a todos los autores participantes. 


\section{Aviso de derechos de autor/a}

La revista Katharsis autoriza la copia de artículos y textos para fines de uso académico o interno de las instituciones citando la fuente. Para la reproducción total o parcial de la revista con otros fines, se debe contar con la autorización explícita de la Institución Universitaria de Envigado.

Las opiniones expresadas por los autores no comprometen a la Institución Universitaria de Envigado.

\section{Dirección de envío}

Coordinador: Olena Klimenko

Correo electrónico: Katharsis@iue.edu.co

Teléfonos: 3391010 ext 223

Página Web: http://revistas.iue.edu.co/index.php/katharsis/about

\section{Referencias consultadas}

Comité de ética para publicaciones (COPE) (2006). Directrices sobre buenas prácticas para publicaciones. [Online]. Disponible en http://www.uta.cl/ddinoticias/adjunto/2014_11.pdf

ICMJE (2015). Recommendations for the Conduct, Reporting, Editing, and Publication of Scholarly Work in Medical Journals. [Online] Disponible en http://www.icmje.org/icmje-recommendations.pdf 